\title{
Orientationally correlated colloidal polycrystals without long-range positional order
}

\author{
Cristina Arcos, ${ }^{1,2}$ Kitty Kumar, ${ }^{1}$ Wenceslao González-Viñas, ${ }^{3}$ Rafael Sirera, ${ }^{2}$ Kristin M. Poduska, ${ }^{1}$ and Anand Yethiraj ${ }^{1}$ \\ ${ }^{1}$ Department of Physics and Physical Oceanography, Memorial University, St. John's, NL, Canada A1B 3X7 \\ ${ }^{2}$ Department of Chemistry and Soil Science, Universidad de Navarra, Pamplona, Spain \\ ${ }^{3}$ Department of Physics and Applied Mathematics, Universidad de Navarra, Pamplona, Spain \\ (Received 16 January 2008; revised manuscript received 31 March 2008; published 22 May 2008)
}

\begin{abstract}
We probe the local and global structure of spin-coated colloidal crystals via laser diffraction measurements and scanning electron and atomic force microscopies, and find that they are unique three-dimensional orientationally correlated polycrystals, exhibiting short-range positional order but long-range radial orientational correlations, reminiscent of - but distinct from—two-dimensional colloidal hexatic phases. Thickness and symmetries are controllable by solvent choice and spin speed. While the polycrystallinity of these colloidal films limits their applicability to photonics, we demonstrate their feasibility as templates to make crack-free magnetic patterns.
\end{abstract}

DOI: 10.1103/PhysRevE.77.050402

PACS number(s): 82.70.Dd, 64.70.pv, 64.75.Yz

The self-assembly of colloidal microspheres has been used to address the fundamental questions of how materials crystallize [1-6] or fail to crystallize [7-9]. Micrometer-scale colloidal crystals can be used as a template that, using further processing methods, can be used to create photonic materials [10-12], optical sensors [13], and antireflection coatings [14]. However, the high density of missing-sphere defects and cracks in photonic crystals produced via self-assembly [15] remains a serious limitation, and thus the study of colloidal defects [16] is an active area of research. Spin-coating of colloidal suspensions is the quickest and most reproducible method to make large-area colloidal crystals. While spin-coating has been proposed to fabricate single crystals for photonic applications $[17,18]$, the symmetric radial optical interference patterns observed are unexpected for single crystals. We find here that spin-coated colloidal films are indeed neither single crystals nor powder polycrystals, but are in fact a unique polycrystal phase. While true singledomain sizes are $\sim 10 \mu \mathrm{m}$, there is orientational correlation on the centimeter scale. Our results demonstrate a novel crystal packing strategy by which long-range orientational order develops in the absence of long-range positional order, reminiscent of two-dimensional colloidal hexatic phases $[19,20]$, and leading to crack-free crystals. Distinct from colloidal hexatic phases, our polycrystals exhibit centimeterscale orientational order, which arises due to the spinning axis and can be produced with fourfold, sixfold, or mixed symmetries for a range of thicknesses as a function of spin speed. The electrodeposition of magnetic material through colloidal polycrystals demonstrates their feasibility for material templating applications.

The standard technique to make large-area close-packed crystals is controlled (vertical) drying, utilizing capillary forces [21-23] to direct self-assembly. Other external shear [24], electric [25], electrohydrodynamic [26,27], and gravitational forces [28] have also been used. Making dried colloidal crystals with these methods is slow, taking from hours to days. Spin-coating has been shown to be a robust technique $[17,18,29,30]$ to make large-area colloidal crystals in minutes. In this work, we correlate measurements of largescale ( $\mathrm{mm}$ and $\mathrm{cm}$ scale) order with local ( $\mu \mathrm{m}$ scale) order to elucidate the structure of spin-coated colloidal crystals.
Evaporative colloid spin-coating consists of discharging colloidal fluid on a substrate, followed by simultaneously spinning-induced fluid spreading and drying. Suspensions of silica spheres $(5 \mathrm{~mL}, 20 \%$ by volume, $458 \pm 2 \mathrm{~nm}$ diameter) were prepared with volatile solvents (ethanol or acetone) by ultrasonicating until opalescent reflections were seen near the edges of the container at $27^{\circ} \mathrm{C}$. The substrates (22 $\mathrm{mm} \times 30 \mathrm{~mm}$ microscope cover slides) were cleaned with $\mathrm{H}_{2} \mathrm{SO}_{4}$ and rinsed thoroughly with ultrapure water prior to use and glued to a microscope slide for structural strength. Seconds after commencing spin-coating, intense colors emanating radially from the center of symmetry were observed when the sample was illuminated with diffuse white light. The symmetry of the optical reflections was fourfold for acetone or sixfold for ethanol samples [Figs. 1(a) and 1(b)]. Single-particle resolution images of the colloidal crystal surfaces, obtained via scanning electron microscopy (SEM) [Figs. 1(c) and 1(d)], and spatially resolved laser diffraction studies using a 1.2-mm-width spatially filtered $(405 \mathrm{~nm}$, $25 \mathrm{~mW}$ ) laser beam operated without any focusing optics [Figs. 1(a) and 1(b)], show particle packings that are consistent with the symmetry of the optical reflections. The nearest-neighbor spacing obtained from laser diffraction was $464 \pm 4 \mathrm{~nm}$ (diffraction grating used for calibration) and $458 \pm 2 \mathrm{~nm}$ by SEM calculated from distances between several touching neighbors in a close-packed crystalline region (since no calibration standard was used, possible systematic errors are $\pm 5 \%$ ). The symmetry of spin-coating results in crack-free crystals, in contrast with spin-coating under identical conditions on a substrate corrugated with parallel lines that breaks center-of-spinning symmetry [Figs. 1(e) and $1(f)]$. Other crystal-growth methods also typically produce crystals with cracks between grains (e.g., in dipcoating, cracks appear at $\sim 50$ sphere diameters [15]).

The presence of four-arm or six-arm crosses across the entire sample suggests a high degree of order. This has been interpreted as a globally even distribution of hexagonally packed spheres [17]. We show this to be incorrect by comparing laser diffraction patterns ( $4 \mathrm{~mm}$ from the sample on a paper-backed screen that autofluoresced in violet light, obtained with a monochrome CCD camera equipped with a $0.3-1 \times$ macro lens) upon continuous translation of a 1.2- 


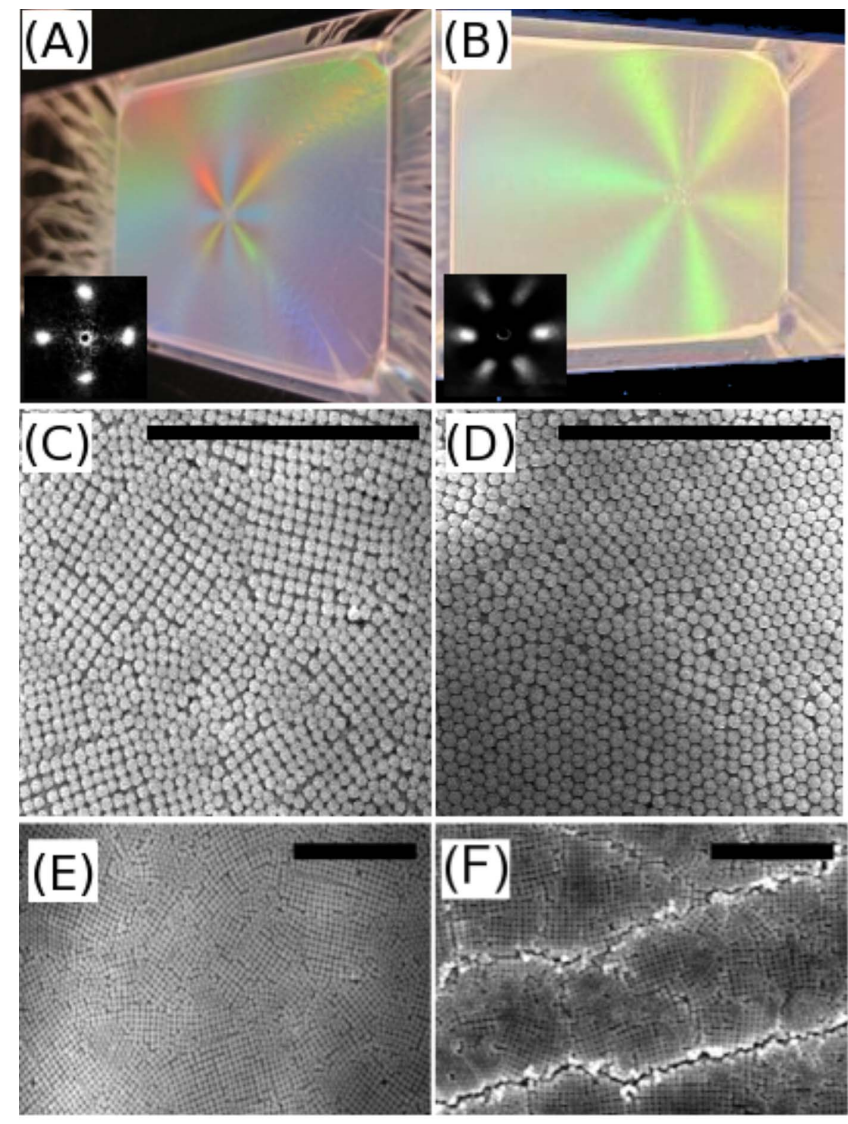

FIG. 1. (Color online) White-light reflections of colloids spincoated at $3000 \mathrm{rpm}$ from (a) acetone and (b) ethanol solvents onto $22 \times 30 \mathrm{~mm}^{2}$ coverslides display fourfold and sixfold symmetry. Laser diffraction patterns (insets) show that the fourfold and sixfold symmetries correspond to fcc (100) and (111) planes with a nearestneighbor spacing $464 \pm 4 \mathrm{~nm}$. (c),(d) SEM images near the center of samples with four-arm and six-arm crosses show planes with square and hexagonal symmetry. (e) Spin-coating produces largearea, crack-free colloidal films. (f) Breaking this symmetry by spincoating onto a substrate corrugated by lines spaced $0.5 \mathrm{~mm}$ apart (there is no line in the field of view) reintroduces cracks. All scale bars are $10 \mu \mathrm{m}$.

mm-diam laser beam along radial lines [Fig. 2(a), R arrow] and off-center chords [Fig. 2(a), O arrow] across the sample. If the sample were single-crystalline, then translation in any direction would leave the peak orientations unchanged, while for a polycrystalline powder the peak orientations would change abruptly at grain boundaries. Instead, we found that the orientations of first-order diffraction peaks were unaffected during radial translation from the center, but rotated continuously when translated off-center in a straight line [Fig. 2(b)]. Angular correlations can be visualized by rotating the observed diffraction patterns in order to bring them into registry with the $x=0$ pattern [Figs. 2(b) and 2(c)]. With the spinning center at $(0,0)$, the diffraction pattern for any laser spot position $(x, y)$ on the sample [with polar coordinates $(r, \alpha)]$ was rotated with respect to that at a reference point $\left(r^{\prime}, \alpha^{\prime}\right)$ by an angle $\left|\alpha-\alpha^{\prime}\right|$. A structure consistent with these observations [Fig. 2(a)] is one of small singlecrystalline domains arranged in radial orientational registry:
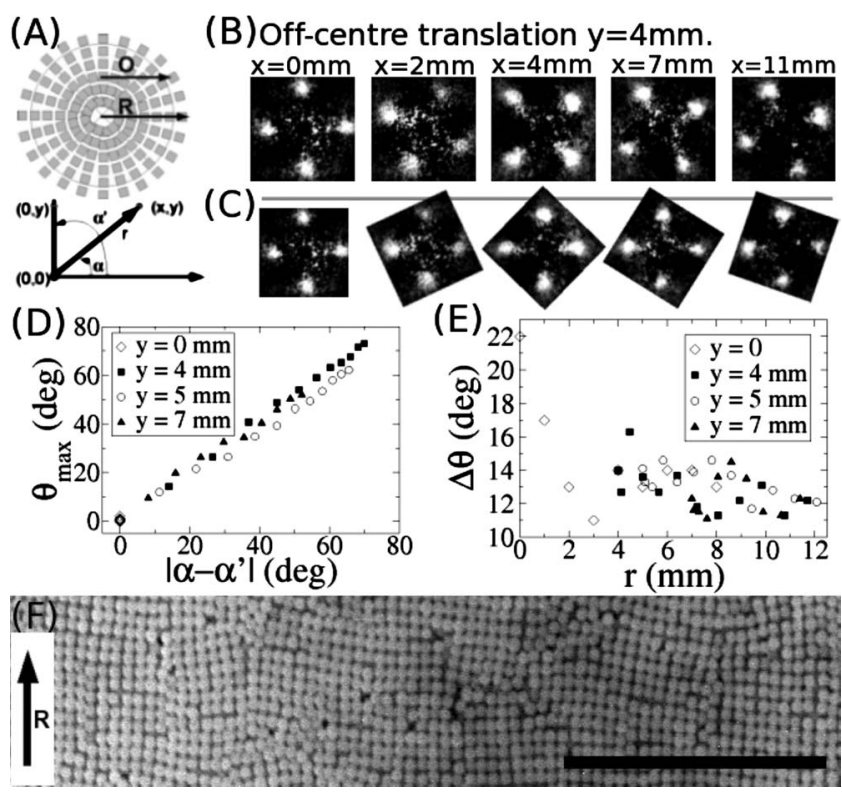

FIG. 2. (a) Proposed structure of the orientationally correlated polycrystal (OCP): gray squares represent small domains in radial orientational registry. Also shown is the axis system and notation used, with the center of spinning at $(0,0)$. (b) Laser diffraction patterns were obtained at $1 \mathrm{~mm} x$ intervals upon radial $(y=0)$ and off-center $(y=4,5$, and $7 \mathrm{~mm})$ translations. Images are shown for $y=4 \mathrm{~mm}$ (acetone, $3000 \mathrm{rpm}$ ). All images obtained along off-center chords-along $\mathrm{O}$ in (a) - show rotation of the laser diffraction pattern while radial translation shows no rotation. (c) Rotation of the diffraction pattern by an angle $\theta_{\max }$ returns the diffraction pattern to the $x=0$ orientation. (d),(e) Correlation analyses for all laser diffraction measurements (at $y=0,4,5$, and $7 \mathrm{~mm}$ ). (d) A plot of $\theta_{\max }$ against $\left|\alpha-\alpha^{\prime}\right|$ (sample orientation relative to the $x=0$ orientation) shows a linear increase with unit slope, consistent with the proposed OCP structure. (e) The domain orientation dispersion $\Delta \theta$ saturates at $12^{\circ}-14^{\circ}$ as a function of radial distance. (f) A tangential section of a typical SEM image (the scale bar is $10 \mu \mathrm{m}$ ) at $(x=0, y=4 \mathrm{~mm})$ shows that true single domains are small but that different domains exhibit small dispersion from the average radial orientation.

we call this an orientationally correlated polycrystal (OCP).

Correlation analyses of laser diffraction images quantify these observations. We first computed the mean value of diffraction intensity $f_{r, \alpha}(\varphi)$ in the radial direction $\rho$ in the vicinity of the first-order laser diffraction peaks as a function of the angle $\varphi$ ( $\rho$ and $\varphi$ are reciprocal space coordinates for the laser diffraction intensity). For crystals with square symmetry, $f_{r, \alpha}(\varphi)$ has four maxima separated by $\Delta \phi \approx 90^{\circ}$. The cross correlation of two diffraction patterns $G\left(\theta ; r, \alpha, r^{\prime}, \alpha^{\prime}\right)=\left\langle f_{r, \alpha}(\varphi) f_{r^{\prime}, \alpha^{\prime}}(\varphi+\theta)\right\rangle_{\varphi}$ (where $\theta$ is the angle by which one of the diffraction patterns is rotated) gives relative orientation between them via the angle $\theta_{\max }$ that maximizes $G$. The correlations are calculated relative to $x=0$ for off-center displacements, and relative to the farthest distance for radial $(y=0)$ displacements. Figure 2(d) shows that for both radial and off-center translation, the rotation angle was consistent with $\theta_{\max }=\left|\alpha-\alpha^{\prime}\right|$, consistent with the structure proposed in Fig. 2(a). The autocorrelation function measured the correlation of a laser diffraction 

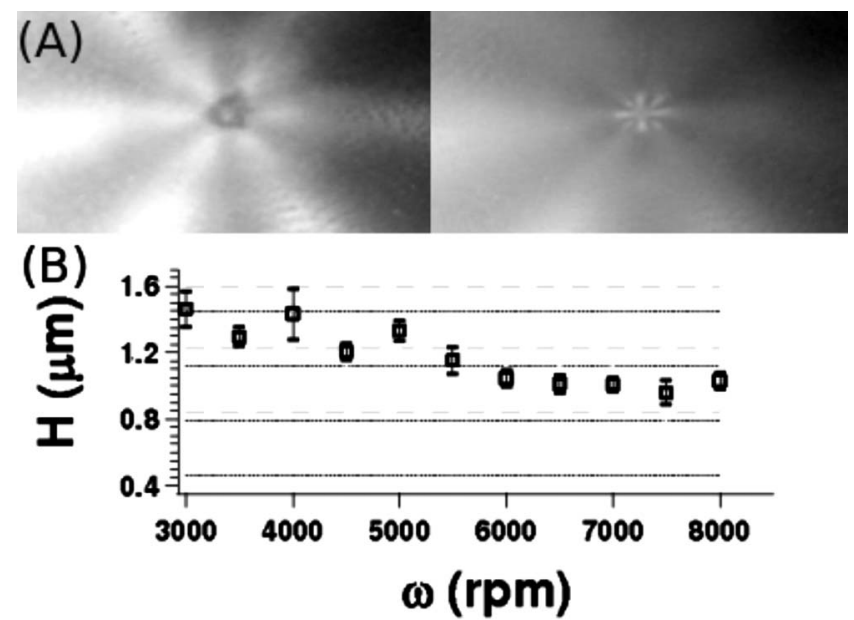

FIG. 3. (a) Red (left) and green (right) channels of a color RGB image of a sample with mixed symmetries: the red channel shows the sixfold symmetry in the bulk of the sample, while the green channel shows the reappearance of fourfold symmetry from the center (colloids in acetone solvent, $7000 \mathrm{rpm}$ ). (b) Sample thickness $H$ (average height of uneven colloid surface, obtained via contactmode AFM) vs $\omega$ displays control of film thicknesses to between two and four layers. Solid and dashed lines are expected thicknesses for integer number (1 to 4) of "square" [fcc (100) face parallel to substrate] and "hexagonal" [fcc (111) face parallel to substrate] structures.

image with itself (but rotated by angle $\theta): G(\theta ; r, \alpha)$ $=\left\langle f_{r, \alpha}(\varphi) f_{r, \alpha}(\varphi+\theta)\right\rangle_{\varphi}$. The domain orientational dispersion $\Delta \theta$ (the half-width of the $\theta=90^{\circ}$ correlation peak at half-height) decreased to a value of $\approx 13^{\circ}$ for radial distances $r>2 \mathrm{~mm}$ [Fig. 2(e)].

The most remarkable property of spin-coated polycrystals is that the long-range (cm-length) orientation correlations shown above coexist with short-range positional correlations. Our observations on disparate length scales are unambiguous. First, SEM images near the sample center [Figs. 1(c) and $1(\mathrm{~d})$ ] and elsewhere [Fig. 2(f)] show typical singlecrystalline regions of $\sim 10 \mu \mathrm{m}$ : true positional order is shortranged. Second, the region probed by the $1.2-\mathrm{mm}$ laser beam contains thousands of micrometer-scale single domains with surprisingly small dispersion in angle from their average value. The domain dispersion $\Delta \theta=13 \pm 1^{\circ}=0.22 \pm 0.03 \mathrm{rad}$ except for regions closest to the center of spinning [Figs. 2(e) and 2(f)]. Indeed, clear diffraction spots are only first observed when the lateral extent of the OCP $(d=r \Delta \theta)$ is comparable to the laser beam spot size; in our experiments this corresponded to $r=2 \mathrm{~mm}$, at which distance the lateral extent of the OCP $(d=r \Delta \theta \sim 0.5 \mathrm{~mm})$ is a large fraction $(\sim 0.4)$ of the laser beam diameter $(1.2 \mathrm{~mm})$. Finally, for distances $r>2 \mathrm{~mm}$ the OCP has long-range (cm scale) radial, but geometrically limited tangential, orientation correlations.

The coexistence of long-range orientational order with short-range positional order is reminiscent of twodimensional phase transitions [31], in particular the colloidal hexatic phase $[19,20]$. However, the OCP structure is distinct from the hexatic phases because long-range ( $\mathrm{cm}$-scale) orientational correlation exists for a wide range of thicknesses. Moreover, we also observe transitions between fourfold and

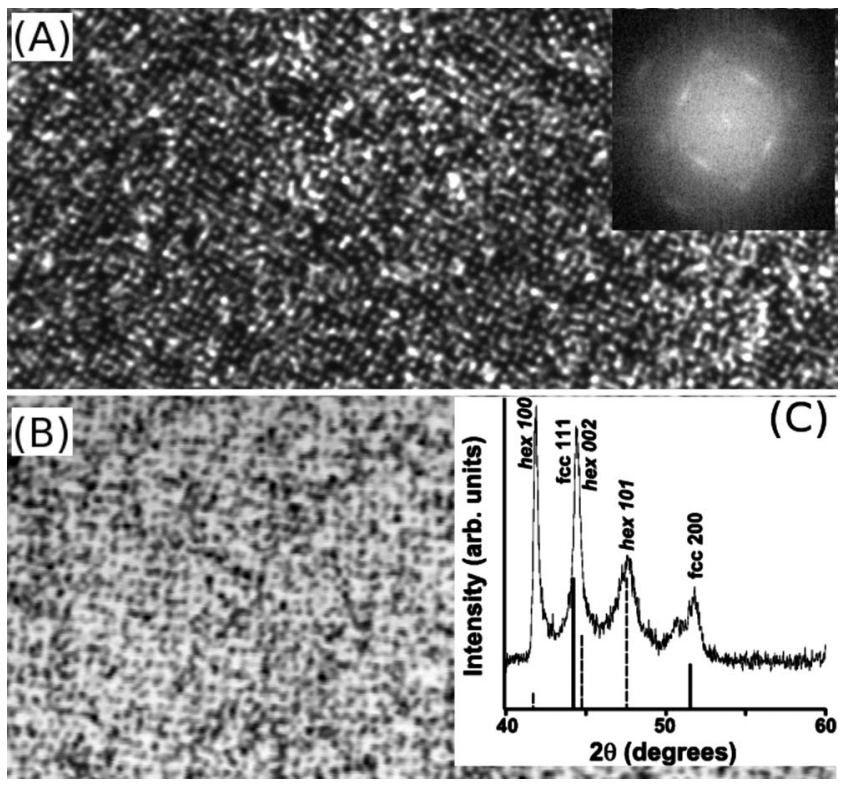

FIG. 4. (a) Transmission optical micrograph, showing cobalt (dark regions) filling interstices in the colloid template without destroying fourfold symmetry (FFT of image in the inset). (b) Reflectance confocal micrograph (488 nm laser) allows $z$-sectioned visualization of patterned electrodeposit beneath the colloidal crystal surface. In reflectance, the metallic regions are bright while the lower-reflectivity silica is dark: Fourfold symmetry is clearly preserved. The smallest feature size, a single sphere, is $0.5 \mu \mathrm{m}$. (c) $\mathrm{X}$-ray diffraction plot shows that the cobalt deposited is predominantly hcp Co (JCPDS 5-727) with smaller amounts of fcc Co (JCPDS 15-806) [34].

sixfold symmetry as a function of angular velocity, with mixed symmetries at the transition, where single samples with multiple symmetries as a function of the distance from the spinning center can be created. Figure 3(a) shows the two channels of a color RGB image: the red channel (left) shows sixfold symmetry in most of the sample, while the green channel (right) shows the reappearance of fourfold symmetry in the center. Samples shown in this work are two to four layers thick [Fig. 3(b)]. Mean colloidal crystal thickness (obtained by contact-mode AFM line scans) decreases as the speed of rotation increases [Fig. 3(b)]. Similar OCPs were also obtained with ten-layer-thick samples (data not shown).

Domain size is crucial to many materials science applications. For photonics, the defect density in colloidal crystals is usually unacceptably large and we see here that spin-coated templates are no improvement. A more forgiving application is to use colloidal templates to make magnetic patterns [32], with potential applications as economical lithography for magnetic data storage materials [33]. Colloidal templates were spin-coated on (111)-textured $\mathrm{Au} / \mathrm{Cr} /$ glass. Cobalt metal was then electrodeposited from an aqueous $0.1 \mathrm{M}$ $\mathrm{CoSO}_{4}$ electrolyte using a constant applied potential $(-1.10 \mathrm{~V}$ vs $\mathrm{Ag} / \mathrm{AgCl}$ reference electrode) in a standard three-electrode cell. Optically thick films $(>20 \mathrm{~nm})$ of cobalt metal formed within $10 \mathrm{~s}$ and $\mu \mathrm{m}$-thick films formed in minutes. Transmission optical microscopy [Fig. 4(a)] confirmed 
that the electrodeposit is dense, uniform, and did not substantially disrupt colloidal order, while a $z$-sectioned reflectance confocal micrograph [Fig. 4(b)] revealed a patterned electrodeposit just beneath the colloidal crystal surface. X-ray diffraction using $\mathrm{Cu} K \alpha$ radiation [Fig. 4(c)] showed predominant hep Co with smaller amounts of fcc Co. Finally, electrodeposits peeled from the substrate were attracted to a permanent magnet indicating ferromagnetic behavior. Thus, electrodeposition of magnetic material through a spin-coated colloidal template does not distort OCP structure and preserves its crack-free nature.

This work was supported by the Natural Science and Engineering Research Council of Canada, and partly by the Spanish MEC (MAT2003-02369, FIS2007-66004-C02-01). K.M.P. and A.Y. acknowledge discussions with Martin Plumer. C.A. acknowledges partial support from the Asociación de Amigos de la Universidad de Navarra.
[1] Z. Cheng, P. Chaikin, J. Zhu, W. Russel, and W. Meyer, Phys. Rev. Lett. 88, 015501 (2001).

[2] J. L. Harland and W. van Megen, Phys. Rev. E 55, 3054 (1997).

[3] U. Gasser, E. R. Weeks, A. Schofield, P. N. Pusey, and D. A. Weitz, Science 292, 258 (2001).

[4] T. Palberg, J. Phys.: Condens. Matter 11, R323 (1999).

[5] A. Yethiraj and A. van Blaaderen, Nature (London) 421, 513 (2003).

[6] A. Yethiraj, Soft Matter 3, 1099 (2007).

[7] W. K. Kegel and A. van Blaaderen, Science 287, 290 (2000).

[8] E. R. Weeks, J. C. Crocker, A. C. Levitt, A. Schofield, and D. A. Weitz, Science 287, 627 (2000).

[9] K. N. Pham et al., Science 296, 104 (2002).

[10] A. Blanco et al., Nature (London) 405, 437 (2000).

[11] Y. A. Vlasov, X-Z. Bo, J. C. Sturm, and D. J. Norris, Nature (London) 414, 289 (2001).

[12] P. V. Braun and P. Wiltzius, Curr. Opin. Colloid Interface Sci. 7, 116 (2002).

[13] J. H. Holtz and S. A. Asher, Nature (London) 389, 829 (1997).

[14] K. C. Krogman, T. Druffel, and M. K. Sunkara, Nanotechnology 16, S338 (2005).

[15] V. L. Colvin, MRS Bull. 26, 637 (2001).

[16] A. Pertsinidis and X. S. Ling, Nature (London) 413, 147 (2001).

[17] P. Jiang and M. J. McFarland, J. Am. Chem. Soc. 126, 13778 (2004).

[18] A. Mihi, M. Ocaña, and H. Míguez, Adv. Mater. (Weinheim, Ger.) 18, 2244 (2006).

[19] A. H. Marcus and S. A. Rice, Phys. Rev. Lett. 77, 2577 (1996).
[20] K. Zahn, R. Lenke, and G. Maret, Phys. Rev. Lett. 82, 2721 (1999).

[21] P. Jiang, J. F. Bertone, K. S. Hwang, and V. L. Colvin, Chem. Mater. 11, 2132 (1999).

[22] L. M. Goldenberg, J. Wagner, J. Stumpe, B.-R. Paulke, and E. Görnitz, Mater. Sci. Eng., C 22, 405 (2002).

[23] M. Yoldi, C. Arcos, B.-R. Paulke, R. Sirera, W. GonzálezViñas, and E. Görnitz, Mater. Sci. Eng., C 28 (7), 1038 (2008).

[24] R. M. Amos, J. G. Rarity, P. R. Tapster, T. J. Shepherd, and S. C. Kitson, Phys. Rev. E 61, 2929 (2000).

[25] A. Yethiraj, J. H. J. Thijssen, A. Wouterse, and A. van Blaaderen, Adv. Mater. (Weinheim, Ger.) 16, 596 (2004).

[26] M. Trau, D. A. Saville, and I. A. Aksay, Langmuir 13, 6375 (1997).

[27] M. Yoldi, W. González-Viñas, M. C. Arcos, and R. Sirera, J. Mater. Sci. 41, 2965 (2006).

[28] J. P. Hoogenboom, P. Vergeer, and A. van Blaaderen, J. Chem. Phys. 119, 3371 (2003).

[29] T. J. Rehg and B. G. Higgins, AIChE J. 38, 489 (1992).

[30] T. Ogi, L. B. Modesto-López, F. Iskandar, and K. Okuyama, Colloids Surf., A 297, 71 (2007).

[31] For a review, see D. R. Nelson, in Phase Transitions and Critical Phenomena, edited by C. Domb, and J. L. Lebowitz (Academic, London, 1983), Vol. 7.

[32] W. Liu, W. Zhong, L. J. Qiu, L. Y. Lu, and Y. W. Du, Eur. Phys. J. B 51, 501 (2006).

[33] B. D. Terris and T. Thomson, J. Phys. D 38, R199 (2005).

[34] Joint Commission on Powder Diffraction StandardsInternational Center for Diffraction Data, Powder Diffraction File (Newtown Square, PA, 2003). 